\title{
Conhecimento das mulheres idosas sobre doenças sexualmente transmissíveis, conhecimento, uso e acesso aos métodos preventivos
}

\author{
Elderly women's knowledge regarding sexually transmitted diseases, and their use and access to preventive measures
}

Conocimiento de mujeres ancianas sobre enfermedades de transmisión sexual; conocimiento, uso y acceso a métodos preventivos

\author{
Tamires Machado Moreira ${ }^{1}$, Bibiane Dias Miranda Parreira², Marina Aleixo Diniz ${ }^{3}$, Sueli Riul da Silva ${ }^{4}$
}

\section{RESUMO}

O presente trabalho trata-se de estudo descritivo, transversal e quantitativo, que teve como objetivos verificar o conhecimento e ocorrência de doenças sexualmente transmissíveis (DST) e o acesso ao tratamento entre mulheres de uma Unidade de Atenção ao Idoso e o entendimento, uso e acesso aos métodos preventivos. A coleta de dados foi realizada por meio de um formulário estruturado com 210 idosas na Unidade de Atenção ao Idoso de Uberaba-MG, e analisada por estatística simples. Identificou-se que $74,8 \%$ das mulheres sabiam o que são DST, sendo a mais conhecida a Síndrome da Imunodeficiência Adquirida (94,8\%); 12,4\% relataram ocorrência de DST, sendo a gonorreia a mais citada (23\%). Os sinais e sintomas mais mencionados foram coceira vaginal $(65,2 \%)$ e corrimento $(57,6)$. A camisinha foi referida como principal método preventivo $(80,5 \%)$. Contudo, somente $20,6 \%$ das sexualmente ativas relataram a sua utilização. Ressalta-se a importância de ações educativas voltadas às DST e métodos preventivos para essa população específica.

Descritores: Doenças Sexualmente Transmissíveis; Saúde do Idoso; Enfermagem.

\section{ABSTRACT}

This descriptive, cross-sectional, quantitative study was performed with the objective to verify the knowledge and occurrence of sexually transmitted diseases (STD) and access to treatment among female clients of an Elderly Health Center and their understanding, use and access to preventive methods. Data collection was performed using a structured form with 210 aged women at the Uberaba Elderly Health Center (Uberaba, MG) and analyzed using simple statistics. It was found that $74.8 \%$ of the women knew what STD were, and the most familiar diseases was the Acquired Immunodeficiency Syndrome (94.8\%); $12.4 \%$ reported having had a STD, and gonorrhea was the most reported (23\%). The signs and symptoms most often mentioned were vaginal itching (65.2\%) and discharge (57.6). The condom was referred as the main prevention method (80.5\%). However, only $20.6 \%$ of the sexually active women referred using condoms. It is highlighted that it is important to implement educational activities regarding STD and preventive methods aiming at this specific population.

Descriptors: Sexually Transmitted Diseases; Health of the Elderly; Nursing.

\section{RESUMEN}

Estudio descriptivo, transversal, cuantitativo, objetivando verificar el conocimiento y ocurrencia de enfermedades de transmisión sexual (DST, en portugués) y la accesibilidad al tratamiento entre mujeres de una Unidad de Atención al Anciano, así como verificar conocimientos, uso y acceso a métodos preventivos. Datos recolectados mediante formulario estructurado con 210 ancianas en la Unidad de Atención al Anciano de Uberaba-MG, analizados por estadística simple. Se determinó que 74,8\% de las mujeres sabían lo que son las DST, resultando la más conocida el Síndrome de Inmunodeficiencia Adquirida (94,8\%); $12,4 \%$ informaron ocurrencia de DST, la más citada fue la gonorrea (23,0\%). Los signos y síntomas más mencionados fueron: picazón vaginal $(65,2 \%)$ y secreción (57,6\%). El preservativo fue sindicado como principal método preventivo $(80,5 \%)$; no obstante, solamente $20,6 \%$ de las sexualmente activas refirieron su utilización. Se resalta la importancia de acciones educativas enfocadas en DST y métodos preventivos para esta población específica.

Descriptores: Enfermedades de Transmisión Sexual; Salud del Anciano; Enfermería.

\footnotetext{
1 Acadêmica do curso de graduação em Enfermagem da Universidade Federal do Triângulo Mineiro (UFTM). Uberaba, MG, Brasil. E-mail: tamires mizi@hotmail.com.

${ }^{2}$ Enfermeira, Mestre em Atenção à Saúde. Professora Assistente da UFTM. Uberaba, MG, Brasil. E-mail: bibianedias@yahoo.com.br.

3 Enfermeira, Mestre em Atenção à Saúde. Discente do Programa de Pós-Graduação em Enfermagem Fundamental da Escola de Enfermagem de Ribeirão Preto da Universidade de São Paulo. Ribeirão Preto, SP, Brasil. E-mail: mafmtm@yahoo.com.br.

${ }^{4}$ Enfermeira, Doutora em Enfermagem Fundamental. Professora Associada da UFTM. Uberaba, MG, Brasil. E-mail: sueliriul@terra.com.br.
} 


\section{INTRODUÇÃO}

De acordo com a Política Nacional do Idoso, art. $2^{\circ}$ "Considera-se idoso, a pessoa maior de sessenta anos de idade"(1). Segundo o Instituto Brasileiro de Geografia e Estatística (IBGE), no Brasil a população idosa poderá chegar a mais de 30 milhões de pessoas nos próximos 20 anos, sendo a parcela da população que mais cresce no país (2). As pessoas com 65 ou mais anos de idade em 1970 representavam 3,1\% da população brasileira e em 2050 será de aproximadamente $19 \%{ }^{(3)}$.

Fisiologicamente o idoso possui alterações, que muitas vezes podem ser observadas por meio da lentidão do pulso, do ritmo respiratório, da digestão e assimilação dos alimentos. Além disso, o próprio indivíduo sente a decadência de sua capacidade de satisfação sexual. Entretanto a atividade sexual não desaparece, somente é realizada com menor frequência e intensidade ${ }^{(4)}$.

Essa continuidade pelo interesse sexual durante a terceira idade é um sinal de manutenção das boas condições de saúde, sendo comprovado em estudos que a prática sexual alivia artrites, aumenta a produção de cortisona das glândulas suprarrenais e contribui igualmente para o equilíbrio psíquico ${ }^{(4)}$.

A sexualidade na terceira idade, assim como em qualquer faixa etária, não compreende apenas o ato sexual em si, mas sim o compartilhamento de sentimentos, companheirismo, carinho, vaidade e cuidado com o corpo. Continuar praticando a sexualidade após os 60 anos é um desejo individual de cada um e se desejado, é um exercício que estimula o cotidiano, desde os pequenos gestos, até os mais expressivos ${ }^{(5)}$.

Ponto de grande relevância a ser discutido relacionado a sexualidade, são as doenças sexualmente transmissíveis (DST). Elas são definidas pelo Ministério da Saúde como doenças causadas por vários tipos de agentes, sendo o contato sexual com uma pessoa infectada, sem a utilização de camisinhas feminina ou masculina, a principal forma de transmissão. Apresentase geralmente na forma de corrimento, feridas, verrugas ou bolhas. Algumas possuem tratamento fácil e rápido, de outras só se tratam os sintomas, permanecendo $\operatorname{ativas}^{(6)}$.

De acordo com o Ministério da Saúde, o modo mais seguro de se evitar as DST é utilizar preservativo em todas as relações sexuais, o que juntamente com a realização periódica de consultas com médico e/ou enfermeiro, pela mulher, pode evitar a disseminação dessas doenças( ${ }^{(6)}$.

Estimativas da Organização Mundial da Saúde (OMS) preveem que ocorra cerca de 340 milhões de casos de DST por ano no mundo, sem incluir herpes genital e o Papiloma Vírus Humano (HPV) ${ }^{(7)}$. Considerando-se os casos da Síndrome da Imunodeficiência Adquirida (AIDS) no Brasil, no período de 2009, foram identificados, 1.263 novos casos de AIDS em pessoas com mais de 60 anos $^{(8)}$.

O aumento de novos casos nesta população pode ser devido ao fato de que em décadas atrás, a população idosa atual não adquiriu o hábito de lidar com métodos de prevenção e não se sentem vulneráveis às $\mathrm{DST}^{(9)}$. Além disso, o aumento também se deve a falta de campanhas de prevenção para este grupo, pois os idosos são tidos como assexuados e a sexualidade ainda é cercada de tabus e preconceitos pela sociedade e pelos profissionais de saúde ${ }^{(10)}$.

Percebe-se que o preconceito e a dificuldade de estabelecerem medidas de prevenção são mais graves nessa população que nos outros segmentos populacionais. O problema está focalizado, principalmente, no tabu social relacionado ao sexo nessa idade, onde se imagina uma dessexualização( ${ }^{(9)}$. Não reconhecer os idosos como população de risco, é um fator que contribui para o aumento dos casos de infecção pelo Vírus da Imunodeficiência Humana (HIV) entre as pessoas com 60 anos ou mais ${ }^{(10)}$.

Um dos desafios da prevenção é fazer com que a pessoa idosa perceba sua vulnerabilidade ${ }^{(10)}$. Além disso, é necessário conscientizar os profissionais da saúde de que os idosos também fazem sexo, e como as outras faixas etárias estão vulneráveis às $\mathrm{DST}^{(11)}$.

A importância desta pesquisa está na necessidade de inserir o idoso nas discussões vinculadas às DST. Vários estudos enfocam os conhecimentos das DST em jovens, entretanto, é pequena a discussão em relação à população idosa. Percebe-se também a falta de políticas públicas e ações assistenciais e educativas pelos profissionais de saúde. Para tanto, os objetivos deste estudo foram: verificar o conhecimento e ocorrência de DST, e o acesso ao tratamento entre mulheres de uma Unidade de Atenção ao Idoso (UAI) e verificar o conhecimento, uso e acesso aos métodos preventivos entre mulheres da UAl. 


\section{METODOLOGIA}

Trata-se de um estudo descritivo, transversal, quantitativo, desenvolvido na UAl do município de Uberaba-MG. Esta instituição foi fundada em setembro de 1986, com o intuito de reunir os idosos para realização de uma atenção integral a estes, atuando com promoção e prevenção em saúde, buscando uma melhora na qualidade de vida ${ }^{(12)}$.

A unidade atende a pessoas idosas com atividades recreativas (canto coral, jogos de mesa, baile, bateria), artísticas (pintura em tela, trabalhos manuais), físicas (dança de salão, natação, ginástica, hidroginástica, musculação) e de educação (alfabetização, informática). A unidade conta com cabeleireiros, assistente social, psicólogo, fisioterapeuta, enfermeiro e terapeuta ocupacional.

A coleta de dados ocorreu no período matutino e vespertino durante um mês (de junho a julho de 2010). Participaram 210 mulheres, cadastradas em atividades físicas com frequência regular. Fizeram parte desta pesquisa os sujeitos que preencheram os seguintes critérios de inclusão: ser mulher, ter 60 anos ou mais, participar de ao menos uma atividade física na UAl, ter frequência regular nas atividades. Foram considerados critérios de exclusão: mulheres que não realizavam mais atividade física durante o período de coleta de dados.

Utilizou-se um formulário estruturado, contendo as variáveis socioeconômicas, historia sexual, ginecológica e obstétrica, conhecimento sobre DST, frequência e acesso a tratamento e conhecimento, uso e acesso a métodos preventivos. Este foi aplicado individualmente, em local restrito, antes ou após a realização das atividades físicas oferecidas na UAI. Foram explicados os objetivos desta pesquisa e apresentado o Termo de Consentimento Livre e Esclarecido, o qual foi assinado pelas entrevistadas.

Os dados obtidos foram digitados e armazenados em uma planilha eletrônica no programa $\operatorname{Excel}^{\circledR}$ e, posteriormente transportados para o software Statistical Package for the Social Sciences (SPSS), versão 17.0, para análise descritiva de frequências. Após a análise e interpretação das informações em questão, descreveram-se os resultados por meio de tabelas para a divulgação.

O estudo somente foi realizado após autorização do responsável do UAl e aprovação do Comitê de Ética em Pesquisa da UFTM (CEP-UFTM), por meio do protocolo 1577/2010.

\section{RESULTADOS E DISCUSSÃO}

A idade mínima das idosas foi de 60 anos e máxima de 90 anos, sendo a média 69,7, a mediana 70,0 e o desvio padrão de 5,86. A faixa etária predominante foi de 60 - 70 anos, 48,6\% (102); 46,2\% (97) eram viúvas, $68,1 \%$ (143) residiam com familiares, $40 \%$ (84) trabalhadoras do lar, 65,7\% (137) tinham renda mensal de um salário mínimo e a escolaridade predominante foi de 4 - 8 anos de estudos $44,8 \%$ (94).

A média de escolaridade foi de 3,7 anos, mediana de 4,0 e o desvio padrão de 2,6 , sendo a menor escolaridade zero (analfabeto) e a maior correspondente a 15 anos de estudos.

Observou-se que 31,0\% (65) das mulheres possuíam vida sexual ativa (Tabela 1$)$.

Tabela 1: Distribuição das mulheres com mais de 60 anos frequentadoras da UAl segundo história sexual, ginecológica e obstétrica. Uberaba, MG, 2010.

\begin{tabular}{|c|c|c|}
\hline Variável & $\mathbf{n}$ & $\%^{*}$ \\
\hline \multicolumn{3}{|l|}{ Possuem vida sexual ativa } \\
\hline Sim & 65 & 31,0 \\
\hline Não & 145 & 69,0 \\
\hline \multicolumn{3}{|l|}{ Possuem parceiro fixo } \\
\hline Sim & 85 & 40,50 \\
\hline Não & 125 & 59,50 \\
\hline \multicolumn{3}{|l|}{ Último exame ginecológico (anos) } \\
\hline Nunca realizou o exame & 3 & 1,40 \\
\hline No ano da entrevista & 85 & 40,50 \\
\hline No ano anterior & 70 & 33,30 \\
\hline 2 à 10 anos & 45 & 21,10 \\
\hline A mais de 10 anos & 2 & 1,0 \\
\hline Não se lembram & 5 & 2,40 \\
\hline
\end{tabular}


O aumento do número de pessoas idosas, acarreta o envelhecimento populacional, que vem acompanhado por uma melhora na qualidade de vida, ocasionando mudanças nos comportamentos ligados a sexualidade, influenciando para relações afetivas mais ativas ${ }^{(13)}$.

Merece atenção a verificação da porcentagem de mulheres sexualmente ativas, 31,0\% (65), encontradas nesse estudo e o número das que possuíam parceiros fixos, 40,5\% (85), percebemos que este é mais elevado, indicando que existiam mulheres que possuíam parceiro, mas não mantinham relação sexual. Apenas uma mulher relatou ter parceiros eventuais.

Em pesquisa qualitativa desenvolvida em Duque de Caxias, Rio de Janeiro-RJ com 15 mulheres idosas, os autores verificaram que, analisando a compreensão da sexualidade por parte das entrevistadas, estas foram claras e objetivas, pontuando o namoro e o companheirismo como os ocupantes do lugar do sexo, ou seja, da relação sexual. O sexo e sexualidade são entendidos pela sociedade e pelas idosas entrevistadas como sinônimos. Apesar de relacionarem sexualidade como expressão de carinho e afeto, posicionam sexualidade e relação sexual no mesmo contexto(14).

A respeito da realização do exame ginecológico, $40,5 \%$ (85) delas realizaram este pela última vez durante o ano da pesquisa, sendo que $1,4 \%$ (3) nunca realizou o exame, e $2,4 \%$ (5) não lembraram quando o realizaram pela última vez. O fato de a maioria estar com o exame ginecológico em dia pode explicar-se pela localização da UAl, que se encontra próximo ao Centro de Atenção Integral à Saúde da Mulher (CAISM), o qual possui atendimento ginecológico.

Em relação ao conhecimento sobre DST, 74,8\% (157) relatam saber o que é, havendo conhecimento de mais de uma DST por mulher, sendo as mais conhecidas, entre as $74,8 \%$ que referiram conhecer, HIV/AIDS, 94,8\% (149), gonorreia, $77,6 \%$ (122), sífilis, $57,6 \%$ (90) e HPV, $56,7 \%$ (89).

Algumas das que disseram não saber o que é DST, quando thes foi enumerado alguns tipos de doenças sexualmente transmissíveis, estas mostraram já ter pelo menos ouvido falar de alguma (Tabela 2). 
Tabela 2: Distribuição das mulheres com mais de 60 anos frequentadoras da UAl segundo conhecimento sobre DST, frequência e acesso a tratamento. Uberaba, MG, 2010.

\begin{tabular}{|c|c|c|}
\hline Variável & $\mathbf{n}$ & $\%^{*}$ \\
\hline \multicolumn{3}{|l|}{ Sabe o que é DST } \\
\hline Sim & 157 & 74,8 \\
\hline Não & 53 & 25,2 \\
\hline \multicolumn{3}{|l|}{ DST conhecida } \\
\hline HIV-AIDS & 199 & 94,8 \\
\hline Gonorréia & 163 & 77,6 \\
\hline Sífilis & 121 & 57,6 \\
\hline HPV & 119 & 56,7 \\
\hline Candidíase & 98 & 46,7 \\
\hline Herpes genital & 92 & 43,8 \\
\hline Cancro mole & 75 & 35,7 \\
\hline Tricomoníase & 40 & 19,0 \\
\hline Clamídia & 39 & 18,6 \\
\hline Gardenerella vaginalis (Vaginose bacteriana) & 33 & 15,7 \\
\hline Donovanose & 16 & 7,6 \\
\hline Linfogranuloma venério & 14 & 6,7 \\
\hline Nenhuma & 9 & 4,3 \\
\hline \multicolumn{3}{|l|}{ Sinais e sintomas já ocorridos } \\
\hline Coceira vaginal & 137 & 65,2 \\
\hline Corrimento & 121 & 57,6 \\
\hline Ulcerações/lesões & 44 & 21,0 \\
\hline Verrugas & 17 & 8,1 \\
\hline Nenhum & 54 & 25,7 \\
\hline \multicolumn{3}{|l|}{ Acha que já teve DST } \\
\hline $\operatorname{Sim}$ & 26 & 12,4 \\
\hline Não & 184 & 87,6 \\
\hline \multicolumn{3}{|l|}{ Qual DST já teve } \\
\hline Não sabem informar & 13 & 50,0 \\
\hline Gonorreia & 6 & 23,0 \\
\hline Candidíase & 4 & 15,4 \\
\hline HPV & 3 & 11,5 \\
\hline Herpes Genital & 2 & 7,7 \\
\hline Cancro mole & 1 & 3,9 \\
\hline Sífilis & 1 & 3,9 \\
\hline \multicolumn{3}{|l|}{ Procurou atendimento para tratar DST } \\
\hline Sim & 23 & 88,5 \\
\hline Não & 3 & 11,5 \\
\hline \multicolumn{3}{|l|}{ Local que procurou atendimento } \\
\hline Serviço de saúde & 22 & 95,6 \\
\hline Serviço de saúde e farmácia & 1 & 4,3 \\
\hline \multicolumn{3}{|l|}{ Profissional que a atendeu } \\
\hline Médico & 23 & 100 \\
\hline
\end{tabular}

Obs: três mulheres acham que já tiveram mais de uma DST.

No Brasil de 1998 a 2005, houve um aumento de pessoas que referiram conhecer uma ou mais DST. Sendo as mais conhecidas a AIDS, gonorreia e sífilis ${ }^{(15)}$.

Em estudo realizado com 165 servidores de uma secretaria do Estado de Mato Grosso com 50 anos ou mais de idade, as DST mais citadas foram a AIDS, sífilis, gonorreia, herpes, HPV e hepatite(13).

Quando questionadas sobre sinais e sintomas que podem ser característicos de DST, 74,3\% (156) das idosas relatam a ocorrência de pelo menos um, sendo os mais citados a coceira vaginal, 65,2\% (101), corrimento, $57,6 \%(90)$ e ulcerações/lesões, $21,0 \%$ (33).

Ao serem perguntadas se já tiveram uma DST, apenas $12,4 \%$ (26) responderam que sim. Destas, a gonorreia foi a mais citada, 23,0\% (6), seguida da candidíase, 15,4\% (4). Porém, 50,0\% (13) não souberam informar o nome da doença. O Ministério da Saúde coloca a clamídia e a gonorreia respectivamente, como as DST com maior incidência no Brasil(16). 
Constatou-se que apesar das mulheres referirem sinais e sintomas de DST, $87,6 \%$ (184) referiram acreditar que nunca tiveram uma doença, o que demonstra que algumas mulheres não fazem ligação dos sinais e sintomas, com a presença de DST. Algumas associam essa sintomatologia com alterações do climatério e estágio da vida (Tabela 3). Todavia, sabe-se que algumas doenças como a vaginose bacteriana e a candidíase são infecções endógenas(17).

Tabela 3: Distribuição das mulheres com mais de 60 anos frequentadoras da UAl segundo conhecimento, uso e acesso a métodos preventivos. Uberaba, MG, 2010.

\begin{tabular}{|c|c|c|}
\hline Variável & $\mathbf{n}$ & $\%^{*}$ \\
\hline \multicolumn{3}{|l|}{ Métodos que previnem DST } \\
\hline Camisinha & 169 & 80,5 \\
\hline Coito interrompido & 79 & 37,6 \\
\hline Anticoncepcional & 35 & 16,7 \\
\hline DIU & 16 & 7,6 \\
\hline Laqueadura tubária & 15 & 7,1 \\
\hline Vasectomia & 12 & 5,7 \\
\hline Diafragma & 10 & 4,8 \\
\hline Espermicida & 3 & 1,4 \\
\hline Nenhum dos acima previne & 19 & 9,0 \\
\hline \multicolumn{3}{|l|}{ Utiliza algum método preventivo } \\
\hline Não & 54 & 79,4 \\
\hline Sim & 14 & 20,6 \\
\hline \multicolumn{3}{|l|}{ Local que adquire o método preventivo } \\
\hline Farmácia & 6 & 40,0 \\
\hline UAl & 6 & 40,0 \\
\hline Posto de saúde & 3 & 20,0 \\
\hline \multicolumn{3}{|l|}{ Possuem dificuldade para aquisição do método } \\
\hline Não & 15 & 100 \\
\hline
\end{tabular}

Em um estudo realizado no Brasil, sobre sinais e sintomas associados a DST, observou-se que houve associação entre faixa etária e a ocorrência de DST, obtendo-se índices mais elevados conforme o aumento da faixa etária. A prevalência auto-relatada de DST, tem maiores valores naqueles que relatam ter tido mais parceiros sexuais, possuir maior renda familiar, maior escolaridade, e a predominância é em homens ${ }^{(15)}$.

Entretanto, observa-se que a população desta pesquisa, são mulheres, com menor renda e escolaridade, ainda assim apresentaram altos valores de ocorrência auto-referida de DST.

Em relação a tratamento, $88,5 \%$ (23) das que acharam já ter tido uma DST, procuraram tratamento, sendo evidenciado esse atendimento em serviços de saúde $95,6 \%$ (22) e por médicos $100 \%$ (23). Não foram relatadas dificuldades no acesso (Tabela 3 ).

Em relação aos métodos preventivos, observou-se que as mulheres possuíam conhecimento acerca de prevenção, pois apesar de algumas citarem outros métodos, coito interrompido, $37,6 \%$ (79) e anticoncepcionais, $16,7 \%$ (35) como se fossem métodos preventivos, a maioria, 80,5\% (169) descreve a camisinha como o principal método para se prevenir as doenças sexualmente transmissíveis (Tabela 3 ).

Estudo realizado com 14 idosas participantes de um grupo de uma Unidade de Saúde, no município de Cianorte-PR, $57 \%$ das entrevistadas tinham conhecimentos dos métodos preventivos, entretanto, duas (14\%) não conheciam as formas de evitar as DST e AIDS. Neste mesmo estudo, apenas três (21\%) idosas responderam que já tinham utilizado algum método preventivo $^{(18)}$.

Em pesquisa qualitativa, com seis idosos que participavam de um grupo de uma Unidade Básica de Saúde numa cidade de médio porte do sul do Rio Grande do Sul, alguns dos entrevistados referiram que cuidados como a higiene corporal servem como prática de prevenção às DST e AIDS. O entendimento destes idosos sobre a prevenção encontrava-se distorcido havendo, para isso, a contribuição de fatores socioculturais, juntos a sua história e concepções ${ }^{(11)}$.

No estudo sobre o conhecimento da infecção pelo HIV-AIDS, com 224 idosos, no município de Anápolis-GO, 
os autores identificaram que apesar do grupo apresentar percentual favorável as formas de transmissão, ainda existe desinformação nas respostas. Verificou-se que $62,1 \%$ citaram como forma de transmissão o compartilhamento de sabonetes, toalhas e assentos sanitários, 79,9\% mencionaram picada de mosquito, 58,3\% comida contaminada, $62,3 \%$ talheres, pratos e copos, $24,2 \%$ beijo no rosto, $49,1 \%$ abraço, aperto de mão e $25 \%$ "internar perto" e "sentar perto"(19).

Na presente pesquisa, dentre as sexualmente ativas apenas 20,6\% (13) mantêm relação sexual protegida, utilizando a camisinha como método de prevenção. A maioria $79,4 \%$ (52) não utilizava nenhum método preventivo durante as relações sexuais, e a justificava se da pelo fato de possuírem apenas um parceiro (Tabela 3).

Em outro estudo com 98 idosos que fazem parte de uma instituição que desenvolve programas para melhoria da qualidade de vida de idosos, no município de Curitiba-PR, com relação ao uso da medida de prevenção, 42,8\% afirmaram fazer uso de algum tipo de medida de prevenção e igualmente, 42,8\% disseram não fazer uso e 14,2\% não responderam a pergunta. Interessante destacar que, dentre os que usam, $64,2 \%$ disseram fazer uso da camisinha, $28,5 \%$ não souberam responder e $7,1 \%$ citaram fazer uso de medicação como medida de prevenção(10).

Estudos revelam a prevalência da não utilização de preservativos por essa população( ${ }^{(13,20)}$. Esse fato é preocupante, visto que ocorre um aumento do número de casos de AIDS na faixa etária de 50 a 70 anos, fazendo-se necessário então, tornar os preservativos um hábito nas relações sexuais dos idosos(21). Destaca-se, atualmente, a relação entre a presença de DST e o aumento do risco de infecção pelo HIV(17).

No estudo com servidores de uma secretaria do Estado do Mato Grosso, 92\% dos participantes concordaram que o preservativo previne DST/AIDS, porém observou-se que $78,5 \%$ dos homens e $86,5 \%$ das mulheres não utilizaram o preservativo na última relação sexual(13).

Em estudo realizado com adultos maiores de 50 anos, soropositivos para HIV, residentes em Capivaride Baixo e Imbituba, SC, os autores questionaram a adesão aos preservativos durante as relações sexuais e constataram que mesmo após a descoberta da doença $42,9 \%$ não utilizavam preservativos ${ }^{(20)}$.

Esse fato gera preocupação pois, as pessoas infectadas pelo vírus, não possuem consciência de prevenção do parceiro, o qual muitas vezes não sabe da doença do outro, ocorrendo a disseminação do vírus e, consequentemente, um aumento da contaminação do HIV e outras DST, nesta faixa etária(20). Existe o conhecimento de que o preservativo é de grande importância na prevenção de DST/AIDS, mas uma parcela muito pequena os utiliza(13).

A possibilidade de o idoso ser infectado pelo HIV parece invisível aos olhos da sociedade, e pelos próprios idosos, por não terem a cultura do uso, e as mulheres por, em geral, não poderem engravidar e acharem inútil o uso do preservativo(10).

Foi observado em estudos que a predisposição a não exigência pelo uso do preservativo durante a relação sexual dos idosos, é a confiança no parceiro(13,22). Pedir para que o idoso utilize a camisinha, pode ser considerado como falta de confiança da mulher.

Os parceiros sexuais, principalmente os de maior faixa etária, necessitam ser estimulados ao uso do preservativo, pois podem apresentar resistência, devido a dificuldades no âmbito cultural, proporcionando distúrbios psicossexuais, como o medo dos distúrbios de егеção(23).

Em relação ao acesso, as que utilizavam o preservativo masculino durante as relações, relataram não encontrarem dificuldades de aquisição. Quanto ao local, as mulheres referiram a farmácia 40,0\% (5), a UAI $40,0 \%$ (5) e o posto de saúde $20 \%$ (3). É importante considerar que a UAl, realiza distribuição de preservativos provenientes do Sistema Único de Saúde (SUS). Considera-se salutar esse tipo de prática desenvolvida por este serviço.

Ressalta-se que a aquisição dos preservativos diretamente pelas mulheres só ocorria na UAl, pois nos demais locais, como farmácias e postos de saúde, eram seus parceiros que faziam essa aquisição.

\section{CONCLUSÃO}

Constatou-se por meio deste estudo que as mulheres entrevistadas na UAl, possuíam conhecimento acerca do que são DST e modo de prevenção, mas algumas não associavam sinais e sintomas com a transmissão de DST. 
Entre as participantes sexualmente ativas, observou-se que apesar de terem o conhecimento da vulnerabilidade de se adquirir uma DST em relações desprotegidas, ainda assim não possuem o hábito do uso de preservativos, justificando-se por possuírem parceiros fixos.

Faz-se necessária a criação de políticas públicas que estimulem a promoção da saúde sexual, por meio de ações educativas, exames ginecológicos e distribuição de preservativos, com foco na prevenção das DST, nessa população específica.

\section{REFERÊNCIAS}

1. Lei N. 8.842 de 4 de Janeiro de 1994. Dispõe sobre a Política Nacional do Idoso, cria o Conselho Nacional do Idoso e dá outras providências. Diário Oficial da União (Brasília). 1994 jan 05.

2. Instituto Brasileiro de Geografia e Estatística [Internet]. Brasília: Ministério do Planejamento, Orçamento e Gestão (BR) [cited 2009 dec 06]. Perfil dos Idosos Responsáveis pelos Domicílios no Brasil 2002. Available

from:http://www.ibge.gov.br/home/presidencia/noticias/25072002 pidoso.shtm.

3. Carvalho JAM, Rodrigues-Wong LL. A transição da estrutura etária da população brasileira na primeira metade do século XXI. Cad. Saúde Pública. 2008;24(3):597-605.

4. Netto FLM. Aspectos biológicos e fisiológicos do envelhecimento humano e suas implicações na saúde do idoso. Pensar a Prática. 2004;7:75-84.

5. Catusso MC. Rompendo o silêncio: desvelando a sexualidade em idosos. Revista Virtual Textos \& Contextos. [Internet]. 2005 [cited 2009 nov 30];4:1-19. Available from:

http://revistaseletronicas.pucrs.br/ojs/index.php/Fass/article/viewFil e/996/776

6. Ministério da Saúde [Internet]. Brasília (BR): Departamento de DST, Aids e Hepatites Virais [cited 2009 dec 9]. O que são DST 2009. Available from: http://www.aids.gov.br/pagina/o-que-sao-dst. 7. Ministério da Saúde [Internet]. Brasília (BR): Departamento de DST, Aids e Hepatites Virais [cited 2009 dec 9]. DST em números 2003. Available from: http://www.aids.gov.br/pagina/dst-emnumeros

8. Ministério da Saúde [Internet]. Brasília (BR): Departamento de DST, Aids e Hepatites Virais [cited 2010 mar 25]. Diagnóstico de Idosos 2009. Available from:

http://www.aids.gov.br/pagina/diagnostico-de-idosos

9. Sousa JL. Sexualidade na terceira idade: Uma discussão da AIDS, envelhecimento e medicamentos para disfunção erétil. DST j. bras. doenças sex. transm. 2008;20(1):59-64.

10. Maschio MBM, Balbino AP, Souza PFR, Kalinke LP. Sexualidade na terceira idade: medidas de prevenção para doenças sexualmente transmissíveis e AIDS. Rev Gaúcha Enferm. 2011;32(3):583-589.

11. Laroque MF, Affeldt AB, Cardoso DH, Souza GL, Santana MG, Lange C. Sexualidade do idoso: comportamento para a prevenção de DST/AIDS. Rev Gaúcha Enferm. 2011;32(4):774-80.

12. Caetano ACM, Tavares DMS. Unidade de Atenção ao Idoso: atividades, mudanças no cotidiano e sugestões. Rev. Eletr. Enf. [Internet]. 2008 [cited 2009 dec 05];10(3):622-31. Available from: http://www.fen.ufg.br/revista/v10/n3/v10n3a08.htm.

13. Olivi M, Santana RG, Mathias TAF. Comportamento, conhecimento e percepção de risco sobre doenças sexualmente transmissíveis em um grupo de pessoas com 50 anos ou mais de idade. Rev. Latino-Am Enfermagem. 2008;16(4):679-685.

14. Coelho DNP, Daher DV, Santana RF, Espírito Santo FH. Percepção de mulheres idosas sobre sexualidade: implicações de gênero e no cuidado de enfermagem. Rev. Rene. [Internet]. 2010 [cited 2012 aug
É importante estimular o papel de educador do profissional da saúde, a fim de promover a conscientização e mudança de comportamento dos idosos. Vale ressaltar que os profissionais devem ter uma visão ampla desse grupo etário, considerando os costumes, as crenças, as vivências e os medos dessa população.

É fundamental também, a oferta dos métodos de prevenção nos serviços públicos de saúde, assim como a facilidade do seu acesso e orientações adequadas da forma e importância do uso desses métodos.

19];11(4):163-173. Available from:

http://www.revistarene.ufc.br/vol11n4_pdf/a18v11n4.pdf 15. Bastos FI, Cunha CB, Hacker MA. Sinais e sintomas associados às doenças sexualmente transmissíveis no Brasil. Rev. Saúde Pública. 2008; 42(Supl 1):98-108.

16. Ministério da Saúde [Internet]. Brasília (BR): Departamento de DST, Aids e Hepatites Virais [cited 2010 mar 12]. DST no Brasil 2010. Available from: http://www.aids.gov.br/pagina/dst-no-brasil 17. Ministério da Saúde. Secretaria de Vigilância em Saúde. Programa Nacional de DST e Aids. Manual de Bolso das Doenças Sexualmente Transmissíveis. Brasília (Brasil): Ministério da Saúde;2006.

18. Frugoli A, Magalhães-Júnior CAO. A sexualidade na terceira idade na percepção de um grupo de idosas e indicações para educação sexual. Arq. Ciênc. Saúde UNIPAR. [Internet]. 2011 [cited 2012 aug 18];15(1):85-93. Available from: http://revistas.unipar.br/saude/article/viewFile/3696/2398 19. Pereira GS, Borges Cl. Conhecimento sobre HIV/AIDS de participantes de um grupo de idosos, em Anápolis-Goiás. Esc. Anna Nery. 2010;14(4): 720-725.

20. Bertoncini BZ, Moraes KS, Kulkamp IC. Comportamento sexual em adultos maiores de 50 anos infectados pelo HIV. DST j. bras. doenças sex. transm. [Internet]. 2007 [cited Nov 20];19(3):75-79. Available from: http://www.dst.uff.br//revista19-2-2007/3.pdf 21. Feitoza $A R$, Souza $A R$, Araújo MFM. A magnitude da infecção pelo HIV-AIDS em maiores de 50 anos no município de Fortaleza-CE. DST j. bras. doenças sex. transm. [Internet]. 2004 [cited 2009 nov 15];16(4):32-37. Available from: http://www.dst.uff.br//revista16-42004/6.pdf

22. Laurentino NRS, Barboza D, Chaves G, Besutti J, Bervian SA Portella MR. Namoro na terceira idade e o processo de ser saudável na velhice: recorte ilustrativo de um grupo de mulheres. RBCEH Revista Brasileira de Ciência do Envelhecimento Humano. [Internet]. 2006 [cited nov 10];3(1):51-63. Available from: http://www.upf.br/seer/index.php/rbceh/article/view/57/50 23. Ministério da Saúde. Secretaria de Atenção à Saúde. Departamento de Ações Programáticas Estratégicas. Manual de Atenção à Mulher no Climatério/Menopausa. Brasília (Brasil): Ministério da Saúde;2008.

Artigo recebido em 05/04/2011. Aprovado para publicação em 10/08/2012. Artigo publicado em 31/12/2012. 